\title{
Biological response modifiers - The therapies of the future
}

\author{
MiCHEL G BERGERON, MD, FRCPC
}

A NTIBIOTICS REVOLUTIONIZED MEDICINE IN THE 1940S; THE '90s will see the discovery and development of biological response modifiers (BRMs) which will radically change the management of infectious diseases, cancer and other pathological processes. In contrast to antimicrobial agents which mainly suppress and/or destroy offending pathogens at the site of infection, and anticancer drugs which aim to stop proliferation and neutralize cancer cells, BRMs can optimize and regulate the body's immune defences and cellular responses, replace deficient or absent mediators of host defences and increase host response to vaccines and chemotherapeutic agents (1).

The development of new products such as interleukins, interferons, hematopoietic cytokines or monoclonal antibodies directed at cancer cells or endotoxin would not have been possible without the expertise of multidisciplinary teams which have in the past few decades increased our knowledge of cell physiology, host response and gene control. Rapidly developing molecular biology technology and new ways of making monoclonal antibodies have allowed the industrial production of these new agents (2).

One of the first BRMs, interferon-alpha, a cytokine, was discovered in the early '70s. Cytokines are cellular messengers with multiple functions including the control of cell proliferation and differentiation. Once triggered by a stimulus, they are produced, activated and liberated. Depending on the cytokines (more than 150 are already known), the amount produced and their

Centre Hospitalier de l'Université Laval, 2705, boulevard Laurier, Sainte-Foy, Québec G1V 4G2. Téléphone (418) 654-2705, Fax (418) 654-2715 target, these agents have either positive or deleterious effects on the host. Much needs to be learned about how, why and when many of these cytokines mediate their action. Some cytokines, with potent pharmacological activity, are now being used as BRMs.

About 50 different types of BRMs are presently being investigated and it is estimated that by the end of this millennium, several billion dollars worth of these agents will be sold. BRMs are developing so rapidly that the Food and Drug Administration of the United States brought together, in August 1990, a special review committee to study these agents. The potential clinical applications have to be evaluated carefully, as the effects of these drugs may be multiple and unpredictable. Several of the new agents which are already in use, including erythropoietin, colony stimulating factors and monoclonal antibodies, have improved the quality of life and in some cases increased survival of not only immunodeficient hosts, but also of apparently immunocompetent patients (3).

'Drug-bug' interaction and 'drug-cancer cell' interaction have been the main foci of research in chemotherapy in the past 50 years. 'Drug-host-bug' and/or 'drug-host-cancer cell' interaction will be more and more the focus of modern chemotherapy. BRMs which can either up- or downregulate host response will often have to be used in combination with other chemotherapeutic agents. The type of BRM, the appropriate dose, timing, mode of administration and duration of therapy of these combinations must be investigated and adapted for each disease condition. Several important questions will have to be answered; for example, in the therapy of severe Gram-negative sepsis, should monoclonal antibodies directed against lipopolysac- 




The Scientific World Journal
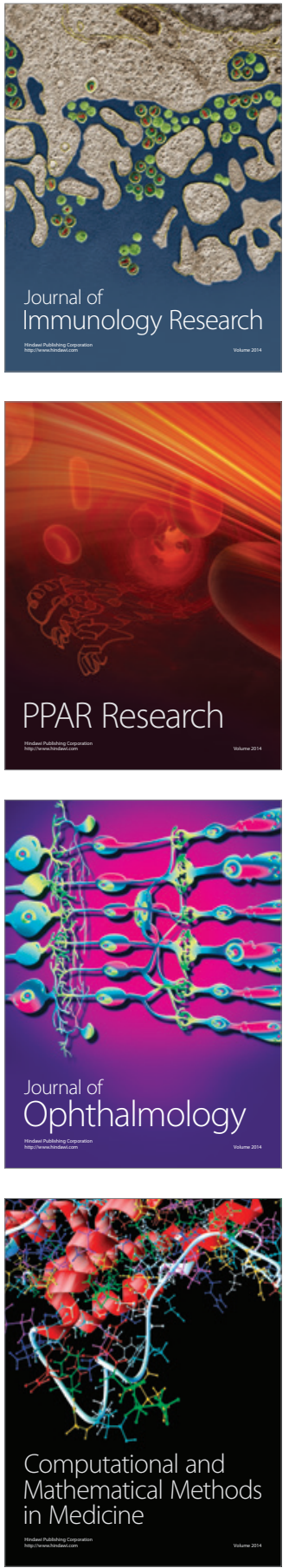

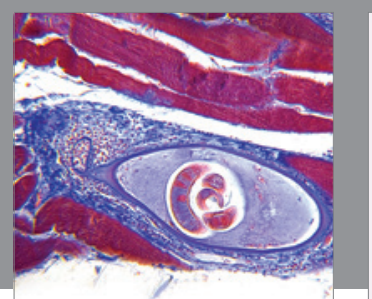

Gastroenterology Research and Practice

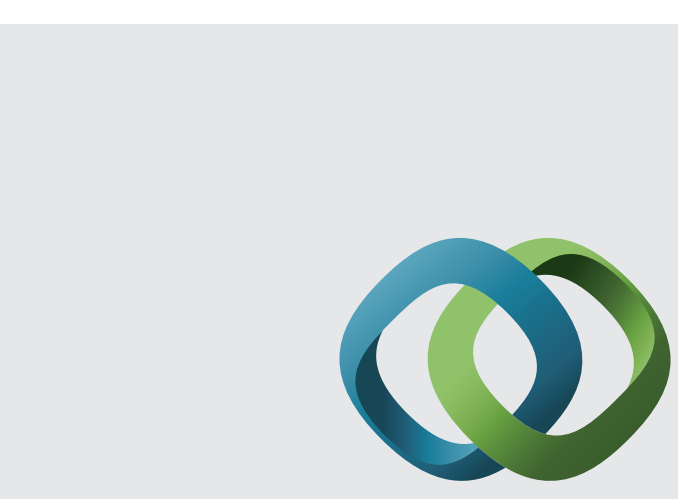

\section{Hindawi}

Submit your manuscripts at

http://www.hindawi.com
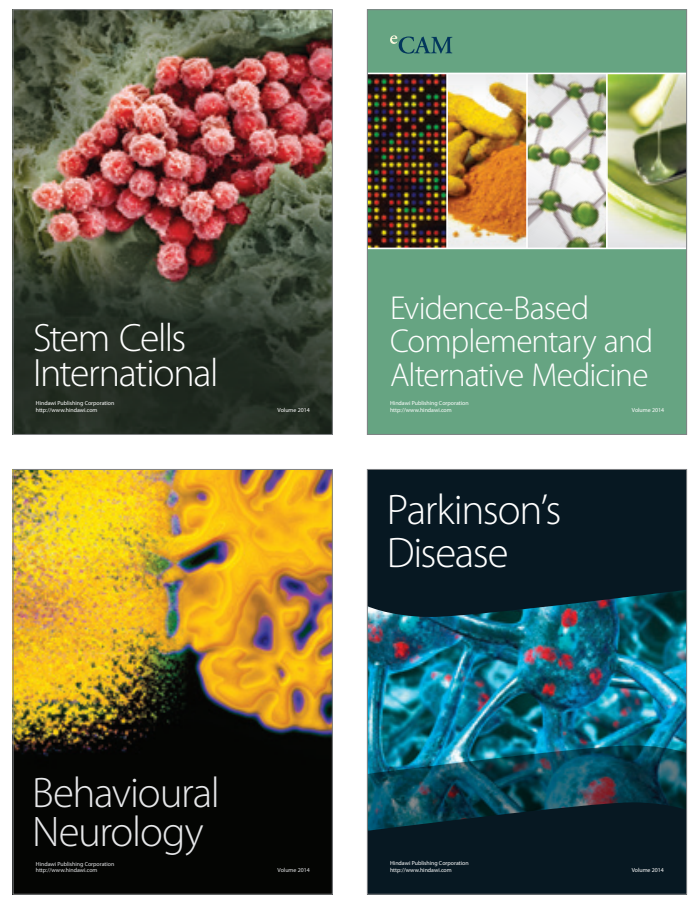
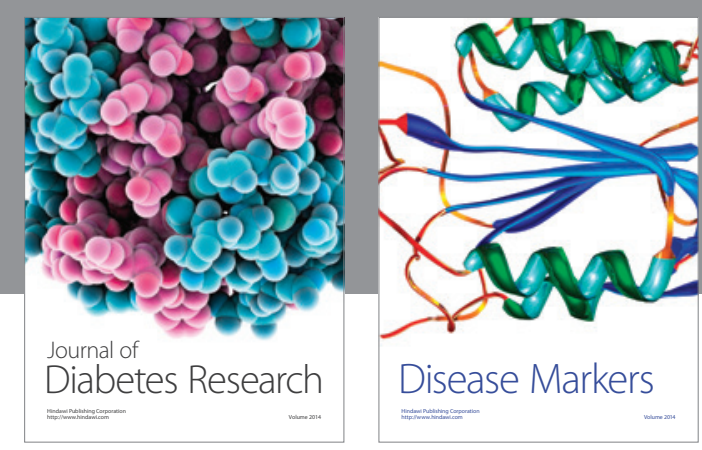

Disease Markers
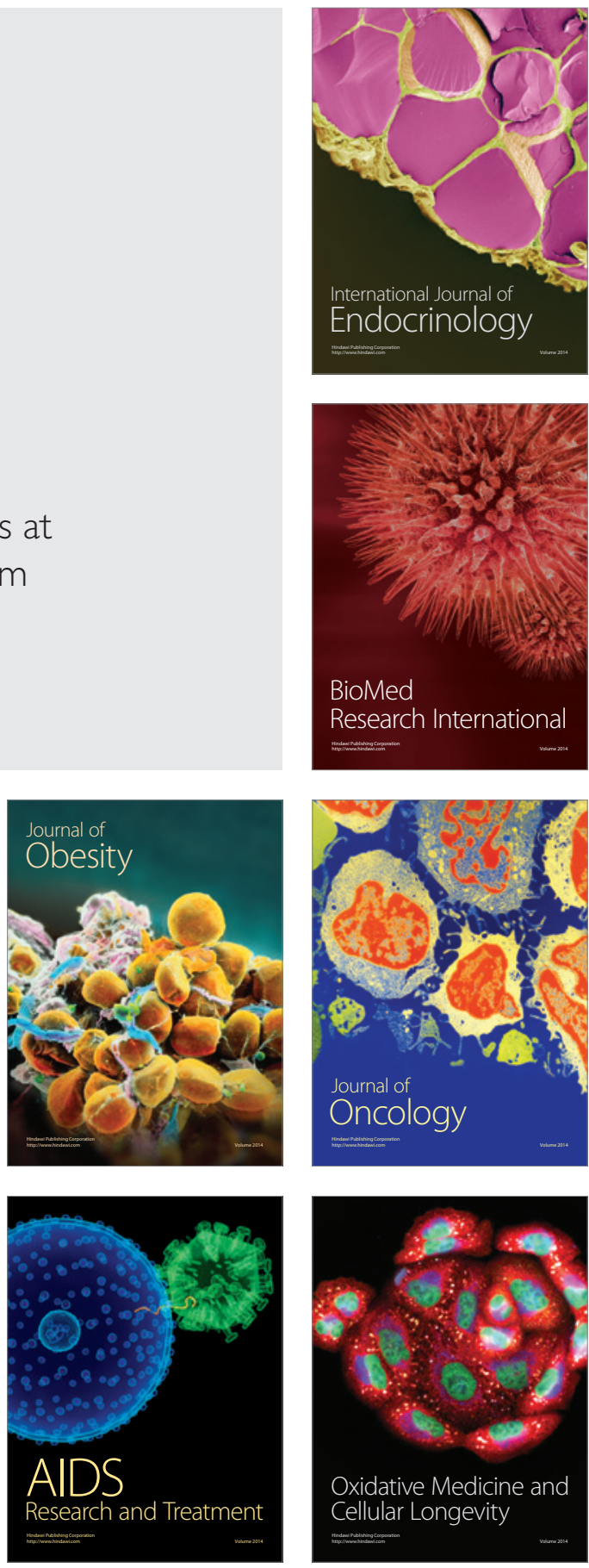